\title{
Stellar evolution models at the Magellanic Cloud metallicities
}

\author{
Raphael Hirschi ${ }^{1,2}$, Sylvia Ekström ${ }^{3}$, Cyril Georgy ${ }^{3}$, \\ Georges Meynet ${ }^{3}$ and André Maeder ${ }^{3}$ \\ ${ }^{1}$ Astrophysics group, Keele University, Lennard-Jones Lab., Keele, ST5 5BG, UK \\ email: r.hirschi@epsam.keele.ac.uk \\ ${ }^{2}$ IPMU, University of Tokyo, Kashiwa, Chiba 277-8582, Japan \\ ${ }^{3}$ Observatoire Astronomique de l'Université de Genève, CH-1290, Sauverny, Switzerland
}

\begin{abstract}
The Magellanic Clouds are great laboratories to study the evolution of stars at two metallicities lower than solar. They provide excellent testbeds for stellar evolution theory and in particular for the impact of metallicity on stellar evolution. It is important to test stellar evolution models at metallicities lower than solar in order to use the models to predict the evolution and properties of the first stars. In these proceedings, after recalling the effects of metallicity, we present stellar evolution models including the effects of rotation at the Magellanic Clouds metallicities. We then compare the models to various observations (ratios of sub-groups of massive stars and supernovae, nitrogen surface enrichment and gamma-ray bursts) and show that the models including the effects of rotation reproduce most of the observational constraints.
\end{abstract}

Keywords. stars: mass loss, stars: rotation, supernovae: general, stars: Wolf-Rayet, galaxies: evolution, Magellanic Clouds, gamma rays: bursts

\section{Introduction}

Massive stars play a key role in the evolution of galaxies via radiative, kinetic and chemical feedback. A current hot topic is the evolution and properties of the first massive stars since these stars took part in the assembly of the first structures in the Universe. The first massive stars died a long time ago and only via simulations can we predict their properties. The simulations can be constrained by observing the chemical signature of these stars in halo low-mass extremely metal-poor stars (Beers \& Christlieb 2005) and by comparing stellar evolution models with observations of massive stars at metallicities lower than solar. The Magellanic Clouds (MCs) are the best place to constrain massive star models since they contain numerous young stars with initial metallicities as low as one fifth solar and they are close enough to observe stars individually. In Sect. 2, we recall the main effects of metallicity. In Sect. 3, we compare models with observations at MC metallicities and conclude in Sect. 4.

\section{The impact of metallicity}

The metallicity $(Z)$ has several effects on the properties of massive stars (see for example Heger et al. 2003; Chieffi \& Limongi 2004; Meynet et al. 1994; Mowlavi et al. 1998). First, low- $Z$ massive stars are more compact due to lower opacity. Second, mass loss is generally weaker at low $Z$. The metallicity $(Z)$ dependence of mass-loss rates is usually described using the formula:

$$
\dot{M}(Z)=\dot{M}\left(Z_{\odot}\right)\left(Z / Z_{\odot}\right)^{\alpha}
$$




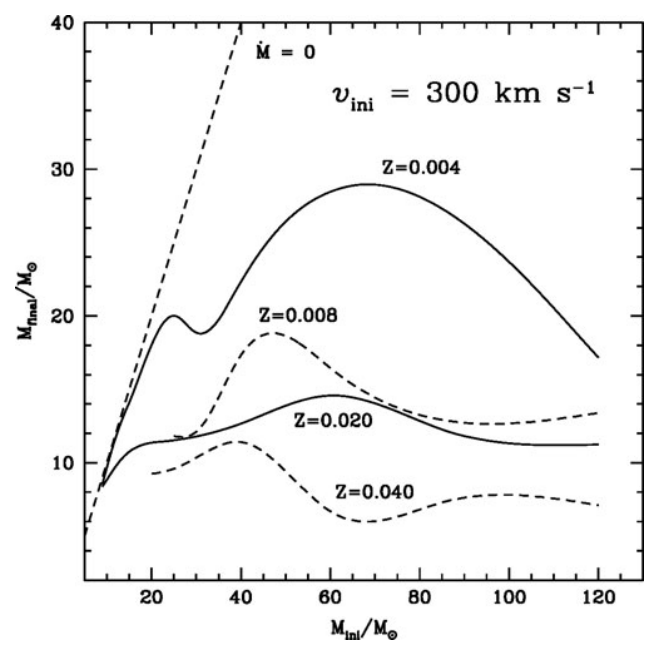

Figure 1. Final mass versus initial mass of models at different metallicities. As the metallicity decreases, mass loss decreases and therefore the final mass increases. At the high mass end, mass loss remains important even at $Z_{\mathrm{SMC}}(\simeq 0.004)$ and the final mass is much smaller than when mass loss is ignored (straight dashed line).

The exponent $\alpha$ varies between 0.5-0.6 (Kudritzki \& Puls 2000, Kudritzki 2002) and 0.7-0.86 (Vink et al. 2001; Vink \& de Koter 2005) for O-type and WR stars respectively (See Mokiem et al. 2007 for a recent comparison between mass-loss prescriptions and observed mass-loss rates). As a result of the weak mass loss at low $Z$, the final mass increases at low $Z$ as shown in Fig. 1 .

Until very recently, most models use at best the total metal content present at the surface of the star to determine the mass-loss rate. However, the surface chemical composition becomes very different from the solar mixture, due either to mass loss in the WR stage or by internal mixing (convection and rotation) after the main sequence. It is therefore important to know the contribution from each chemical species to opacity and mass loss. Recent studies (Vink et al. 2000; Vink \& de Koter 2005) show that iron is the dominant element concerning radiation line-driven mass loss for O-type and WR stars. In the case of WR stars, there is however a plateau at low metallicity due to the contributions from light elements like carbon, nitrogen and oxygen (CNO). In between the hot and cool parts of the HR-diagram, mass loss is not well understood. Observations of the LBV stage indicate that extremely high mass loss may take place during eruptions (see, e.g., Smith et al. 2003) and there is no indication of a metallicity dependence (see for example Pustilnik et al. 2008). In the red supergiant (RSG) stage, the rates generally used are still those of Nieuwenhuijzen \& de Jager (1990). More recent observations indicate that there is a very weak dependence of dust-driven mass loss on metallicity. Van Loon et al. (2005) provide recent mass-loss-rate prescriptions in the RSG stage. From a theoretical point of view, for dust formation, nucleation seed components like silicon and titanium are dominant (van Loon 2000; van Loon 2006; Ferrarotti \& Gail 2006). The ratio of carbon to oxygen is important to determine which kind of molecules and dust form. If the ratio of carbon to oxygen is larger than one, then carbon-rich dust would form, and more likely drive a wind since they are more opaque than oxygen-rich dust at low metallicity (Höfner \& Andersen 2007).

How do rotation induced processes vary with metallicity? The surface layers of massive stars usually accelerate due to internal transport of angular momentum from the core to 
the envelope. Since at low $Z$, stellar winds are weak, this angular momentum dredged up by meridional circulation remains in the star, and the star more easily reaches critical rotation. At the critical limit, matter can easily be launched into a keplerian disk, which probably dissipates under the action of the strong radiation pressure of the star. The efficiency of meridional circulation (dominating the transport of angular momentum) decreases towards lower $Z$ because the Gratton-Öpik term of the vertical velocity of the outer cell is proportional to $1 / \rho$. On the other hand, shear mixing (dominating the mixing of chemical elements) is more efficient at low $Z$. Indeed, the star is more compact and therefore the gradients of angular velocity are larger and the mixing timescale (proportional to the square of the radius) is shorter. This leads to stronger internal mixing of chemical elements at low $Z$ (Meynet \& Maeder 2002).

\section{Comparison between models and observations}

There are many observational constraints that stellar evolution models need to successfully reproduce. In this section, we present models including the effects of rotation and show how they can reproduce several of these constraints.

\subsection{Sub-types of massive stars and supernovae}

The first test is the ratio of subtypes of massive stars and supernovae. Meynet \& Maeder (2005) show that rotating models well reproduce the ratio of WR to O-type stars, which tests the lifetime of the different stages of stellar evolution. The ratio of type Ib or Ic SNe to type II provides constraints on the initial mass ranges of stars finishing their life giving rise to these different supernova types. Prieto et al. (2008) present new observational values for the variation with the metallicity of the number ratio ( $\mathrm{SN} \mathrm{Ib+SN} \mathrm{Ic)/SN} \mathrm{II} \mathrm{to}$ which theoretical predictions can be compared. We discuss here the predictions of single star models for the type Ib/Ic supernovae frequency. Since these supernovae do not show any $\mathrm{H}$-lines in their spectrum, they should have as progenitors stars having removed at least their H-rich envelope by stellar winds, i.e. their progenitors should be WR stars of the WNE type (stars with no $\mathrm{H}$ at their surface and presenting $\mathrm{He}$ and $\mathrm{N}$ lines) or of the $\mathrm{WC} / \mathrm{WO}$ type. Considering that all models ending their lifetime as a WNE or WC/WO phase will explode as a type Ibc supernova, it is possible to compute the variation with the metallicity of the number ratio of type Ibc to type II supernovae.

The result is shown in Fig. 2. One sees that this ratio increases with the metallicity. This is due to the fact that the minimum initial mass of stars ending their life as WNE, or WC/WO stars decreases with increasing metallicity. Single rotating star models can reasonably well reproduce the observed trend with the metallicity. They however give slightly too small values with respect to the observations, and the difference may be explained by the binary stars contribution to the formation of SN Ibc. Models accounting for single and binary channel (but without rotation) are shown as a dotted line (Eldridge et al. 2008). They provide a good fit to the observations. But in that case, most of the supernovae originate from the binary channel, leaving little place for the single star scenario. These models would also predict that most of the WR stars are the outcome of close binary evolution, which is not confirmed by observations (see Foellmi et al. 2003a,b). Most likely, both the single and binary channels contribute. We also computed (SN Ib + SN Ic)/ SN II ratios with the assumption that all models massive enough to form a black hole $(\mathrm{BH})$ do not produce a SN. Comparing the theoretical prediction with this assumption (dashed line in Fig. 2) with the observed rates, we see that in the case no supernova event occurs when a BH is formed, single star models might still account for a significant fraction of the type Ibc supernovae for $Z>0.02$, whereas at low $Z$, no 


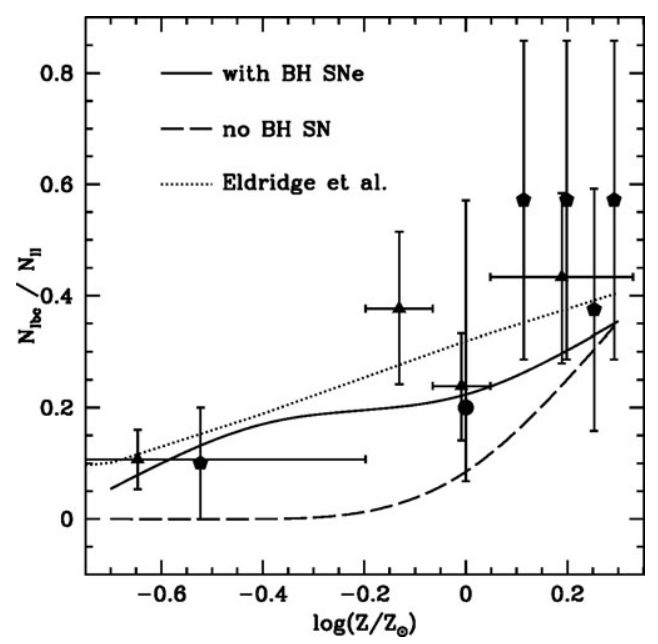

Figure 2. Rate of SN Ibc / SN II if all models produce a SN (solid line) or if models producing a black hole do not explode in a SN (dashed line). Pentagons are observational data from Prieto et al. (2008), and triangles are data from Prantzos \& Boissier (2003). The dotted line represents the binary models of Eldridge et al. (2008).

contribution is expected from single stars. It is however likely that some stars forming black holes produce a SN.

\subsection{Nitrogen surface enrichment}

Surface enrichment in helium and nitrogen are good probes of internal mixing in main sequence stars. While non-rotating models predict no such enrichment before the first dredge-up occurring in the red supergiant stage, rotating models predict enrichment already during the main sequence. This is shown in Fig. 3, where the nitrogen enrichment is plotted along the $\mathrm{z}$ axis and the HR diagram in the $\mathrm{x}-\mathrm{y}$ plane.

The recent FLAMES survey of massive stars (see contribution by Evans) has provided a wealth of data on massive stars (mass loss, initial composition, surface rotational velocities and chemical enrichment, etc.). Hunter et al. (2008) study the correlation between surface nitrogen enrichment and rotation velocities and find that a majority of stars in their sample are consistent with models of single rotating stars. They also find two groups of stars that apparently disagree with the models: 1) a group of fast rotating stars showing little nitrogen enrichment and 2) a group of slowly rotating stars with high nitrogen enrichment. They mention binarity and magnetic fields as possible sources of discrepancies between the observations and the models. The surface enrichment is not only a function of the rotational velocity, it also depends on the mass, the chemical composition, the evolutionary stage and the multiplicity. These observations have recently been re-analysed by Maeder et al. (2008) who show that when the variation of the other intervening parameters is limited, a good agreement between models of single rotating stars and observations is found, and the two discrepant groups disappear or are significantly reduced.

\subsection{Gamma-Ray Bursts progenitors and Oe/Be stars}

Gamma-Ray Bursts have now been firmly connected with type Ic SNe (see Woosley \& Bloom 2006 for a review). Models including the effects of rotation are able to reproduce the frequency and the upper metallicity limit of observed GRBs assuming that only type Ic SNe are able to produce GRBs (Hirschi et al. 2005). However, these models (not 


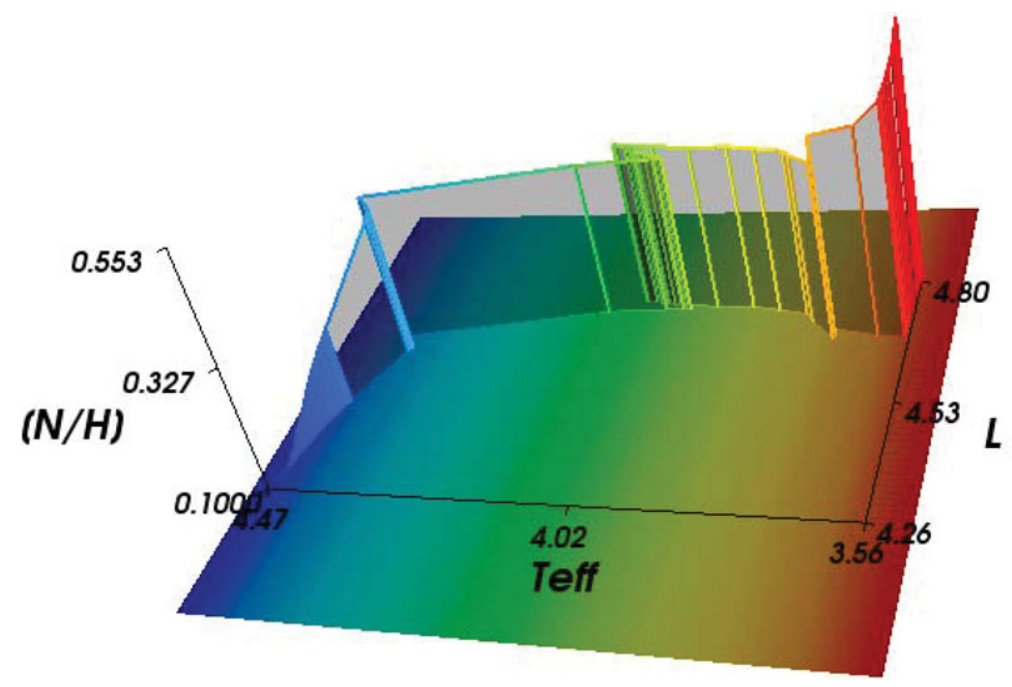

Figure 3. Surface $[\mathrm{N} / \mathrm{H}]$ ratio (z axis) divided by a factor ten along the evolutionary track in the HR diagram (x-y plane) for a rotating $15 \mathrm{M}_{\odot}$ model at solar metallicity. Rotating models predict surface enrichment in nitrogen and helium already on the main sequence whereas non-rotating models predict no enrichment until the first dredge-up.

including the effects of magnetic fields) overestimate the initial rotation rate of pulsars. The inclusion of the effects of magnetic fields according to Spruit (2002) allows a better reproduction of the initial pulsar periods (Heger et al. 2005). Along with gravity waves, magnetic fields are one possible cause for the flat rotation profile of the Sun (Eggenberger et al. 2005). Although it becomes much harder for the core of massive stars to retain enough angular momentum until the core collapse, there is still an evolutionary scenario, the so-called chemically homogeneous evolution, leading to the production of fast rotating cores at the pre-SN stage and therefore enabling MHD explosions and GRBs (see Yoon et al. 2006; Woosley \& Heger 2006). The theoretical GRB event rates obtained by Yoon et al. (2006) are in good agreement with observations apart from the upper metallicity limit, which is lower than the observed one (Modjaz et al. 2008). Although the upper $Z$ limit is still an open issue, it is very likely that critically rotating massive stars like Oe and Be stars are the progenitors of GRBs. Ekström et al. (2008) study in detail the evolution of stars towards the critical limit and theoretical results compare well with observations of Oe/Be stars (see Martayan et al. 2007 and contribution by Martayan in this volume).

\section{Conclusion and outlook}

We have shown that models of massive stars including the effects of rotation are able to reproduce many observable constraints at the Magellanic Cloud metallicities. These models are therefore apt to simulate the evolution of the first massive stars and predict their impact on the early evolution of the Universe. There are still open issues like the upper $Z$ limit for GRBs or the importance of binarity and magnetic fields. Large surveys like the FLAMES survey of massive stars are very useful to quantitatively constrain stellar evolution models and we will certainly learn a lot in the near future. New grids of models are underway at metallicities ranging from solar down to zero in order to predict 
the radiative, kinetic and chemical feedback of massive stars through the ages and to resolve current discrepancies between models and observations.

\section{References}

Beers, T. C. \& Christlieb, N. 2005, ARAA, 43, 531

Chieffi, A. \& Limongi, M. 2004, ApJ, 608, 405

Eggenberger, P., Maeder, A., \& Meynet, G. 2005, A\&SA, 440, L9

Ekström, S., Meynet, G., Maeder, A., \& Barblan, F. 2008, A\&A A, 478, 467

Eldridge, J. J., Izzard, R. G., \& Tout, C. A. 2008, $M N R A S, 384,1109$

Ferrarotti, A. S. \& Gail, H.-P. 2006, A\&SA, 447, 553

Foellmi, C., Moffat, A. F. J., \& Guerrero, M. A. 2003a, MNRAS, 338, 360

Foellmi, C., Moffat, A. F. J., \& Guerrero, M. A. 2003b, MNRAS, 338, 1025

Heger, A., Fryer, C. L., Woosley, S. E., Langer, N., \& Hartmann, D. H. 2003, ApJ, 591, 288

Heger, A., Woosley, S. E., \& Spruit, H. C. 2005, ApJ, 626, 350

Hirschi, R., Meynet, G., \& Maeder, A. 2005, A\&A, 443, 581

Höfner, S. \& Andersen, A. C. 2007, A\& A, 465, L39

Hunter, I., Brott, I., Lennon, D. J., et al. 2008, ApJ, 676, L29

Kudritzki, R.-P. 2002, ApJ, 577, 389

Kudritzki, R.-P. \& Puls, J. 2000, ARAA, 38, 613

Maeder, A., Meynet, G., Ekström, S., \& Georgy, C. 2008, in Comm. in Asteroseismology, Contribution to the Proceedings of the $38^{\text {th }}$ LIAC, in press (arXiv.0810.0657)

Martayan, C., Floquet, M., Hubert, A. M., et al. 2007, A\&A, 472, 577

Meynet, G. \& Maeder, A. 2002, A\& $A, 390,561$

Meynet, G. \& Maeder, A. 2005, A\&\&A, 429, 581

Meynet, G., Maeder, A., Schaller, G., Schaerer, D., \& Charbonnel, C. 1994, A\&AS, 103, 97

Modjaz, M., Kewley, L., Kirshner, R. P., et al. 2008, AJ, 135, 1136

Mokiem, M. R., de Koter, A., Vink, J. S., et al. 2007, A\& A, 473, 603

Mowlavi, N., Meynet, G., Maeder, A., Schaerer, D., \& Charbonnel, C. 1998, A\& $A, 335,573$

Nieuwenhuijzen, H. \& de Jager, C. 1990, A\& A, 231, 134

Prieto, J. L., Stanek, K. Z., \& Beacom, J. F. 2008, ApJ, 673, 999

Prantzos, N. \& Boissier, S. 2003, A\& A, 406, 259

Pustilnik, S. A., Tepliakova, A. L., Kniazev, A. Y., \& Burenkov, A. N. 2008, MNRAS, 388, L24

Smith, N., Gehrz, R. D., Hinz, P. M., et al. 2003, AJ, 125, 1458

Spruit, H. C. 2002, $A \mathscr{E} A, 381,923$

van Loon, J. Th. 2000, $A \& A, 354,125$

van Loon, J. Th. 2006, in H. J. G. L. M. Lamers, N. Langer, T. Nugis, \& K. Annuk (eds.), Stellar Evolution at Low Metallicity: Mass Loss, Explosions, Cosmology, ASP Conf. Ser. 353, p. 211

van Loon, J. Th., Cioni, M. -R. L., Zijlstra, A. A., \& Loup, C. 2005, A\&\&A, 438, 273

Vink, J. S. \& de Koter, A. 2005, A\& $A$, 442, 587

Vink, J. S., de Koter, A., \& Lamers, H. J. G. L. M. 2000, $A \mathscr{E} A$, 362, 295

Vink, J. S., de Koter, A., \& Lamers, H. J. G. L. M. 2001, $A \& \& A, 369,574$

Woosley, S. E. \& Bloom, J. S. 2006, ARAA, 44, 507

Woosley, S. E. \& Heger, A. 2006, ApJ, 637, 914

Yoon, S. -C., Langer, N., \& Norman, C. 2006, A\&广A, 460, 199 